\title{
SYNTHESIS OF NANOCRYSTALLINE DIAMOND FILMS ON MOLYBDENUM SUBSTRATE BY FLAME COMBUSTION METHOD
}

\author{
MAMORU TAKAHASHI \\ Department of Mechanical Engineering, Akita University, 1-1 Tegatagakuen-machi, \\ Akita,010-8502,Japan \\ mtaka@ipc.akita-u.ac.jp \\ MASATAKA SUGAWARA, OSAMU KAMIYA and TADASHI OHYOSHI \\ Department of Mechanical Engineering, Akita University, 1-1 Tegatagakuen-machi, \\ Akita,010-8502,Japan
}

\begin{abstract}
The flame combustion method enables the synthesis of diamond using acetylene-oxygen gas flame combustion in ambient atmosphere. It has various advantages over other methods. In this study, to obtain nanocrystalline diamond films and to achieve good adhesion, diamond films were synthesized by flame combustion using a mixture of commercial or high purity acetylene and oxygen gas with the addition of nitrogen gas. Nitrogen gas added as the nanocrystalline diamond promotion agent; nitrogen flow rate was varied. According to the results, at the mixture of commercial acetylene and oxygen gas, as nitrogen flow rate was increased, the diamond nanocrystallites was high density on the microcrystallites. At the mixture of high purity acetylene and oxygen gas, the optimal nitrogen flow rate was $0.500 \mathrm{~cm}^{3} / \mathrm{s}$, and the diamond nanocrystallites was synthesized continuously on the diamond microcrystallites. They sowed very high density.
\end{abstract}

Keywords: Synthesized nanocrystalline diamond films; Combustion flame; Nitrogen addition.

\section{Introduction}

Owing to its excellent properties, diamond is widely used in the industry. Nanocrystalline diamond has been investigated, due to unique properties of nanocrystalline diamond ${ }^{1}$. Moreover, nanocrystalline diamond film has attracted interests for applications such as in mechanical, electrical and medical fields ${ }^{2}$.

The flame combustion method enables the synthesis of diamond using acetyleneoxygen gas flame combustion in ambient atmosphere ${ }^{3}$. It has various advantages over other methods, such as high synthesis speed, etc. However, during cooling, most diamond films delaminate as a result of thermal stress. We previously synthesized diamond films on a Mo substrate surface by the flame combustion method ${ }^{4-6}$. To prevent diamond film delamination for the synthesis of good diamond films, a three-step synthesis method was proposed $^{5}$ and its optimal conditions were determined ${ }^{5,6}$. 
In this study, to obtain nanocrystalline diamond films and to achieve good adhesion, diamond films were synthesized by flame combustion using a mixture of commercial or high-purity acetylene and oxygen gas with the addition of nitrogen gas. Nitrogen gas added as the nanocrystalline diamond promotion agent; nitrogen flow rate was varied. A three-step synthesis method was used to prevent film delamination. The effects of acetylene gas and nitrogen flow rate on the synthesized films were investigated. Nanocrystalline diamond films and good adhesion were obtained using a mixture of commercial or high-purity acetylene and oxygen gas with nitrogen gas added.

\section{Experimental details}

\subsection{Substrate}

Molybdenum (Mo) of $99.9 \%$ purity was used as the substrate for synthesizing diamond. A Mo rod of $10 \mathrm{~mm}$ in diameter was used; it was cut into disk-shaped fragments of about $3 \mathrm{~mm}$ thickness. As a pretreatment to prevent the delamination, scratch processing, in which a substrate surface is ground with emery paper in one direction, was performed. Furthermore, as growth nuclei for the diamond synthesis, diamond seed particles of about $0.25 \mu \mathrm{m}$ in diameter were dispersed in acetone, the Mo substrate were added, and seed attachment processing was performed for 30 minutes with an ultrasonic syringe.

\subsection{Experimental equipment}

The experimental equipment is shown in Fig. 1 . A $100 \times 100 \times 55 \mathrm{~mm}^{3}$ copper box was used for cooling. Cooling water was poured into this box and the film surface temperature was kept constant. As a support for cooling, a Mo rod of $10 \mathrm{~mm}$ in diameter was set vertically at the center of the box and fixed to a table by a flange. The Mo substrate was attached to this Mo rod. The cooling box was put on a stage. Since it was capable of moving vertically, the distance from the cooling waterside to the film surface was changed, and film surface temperature was controlled. A burner was used for welding. The diameter of the exit of the burner was $1 \mathrm{~mm}$.

\subsection{Three-step synthesis method}

A three-step synthesis method was proposed to prevent film delamination ${ }^{5}$. In this method, the film surface temperature is changed three times during the synthesis. The first step of the method is performed at $1423 \mathrm{~K}$. This was because high bonding strength can be achieved at high temperatures, even though good diamond films are not synthesized. The intermediate layer that works as the buffer phase for thermal stress reduction is synthesized on the Mo substrate. The second step is performed at $1223 \mathrm{~K}$, because a good diamond phase can be synthesized at this temperature. To obtain sufficient thickness of the film, the third step is performed at $1323 \mathrm{~K}$. In the first step, diamond is synthesized, and $\mathrm{Mo}_{2} \mathrm{C}$ is also deposited on the substrate. In the second and third steps, diamond is synthesized. The average thickness of the synthesized film by the 


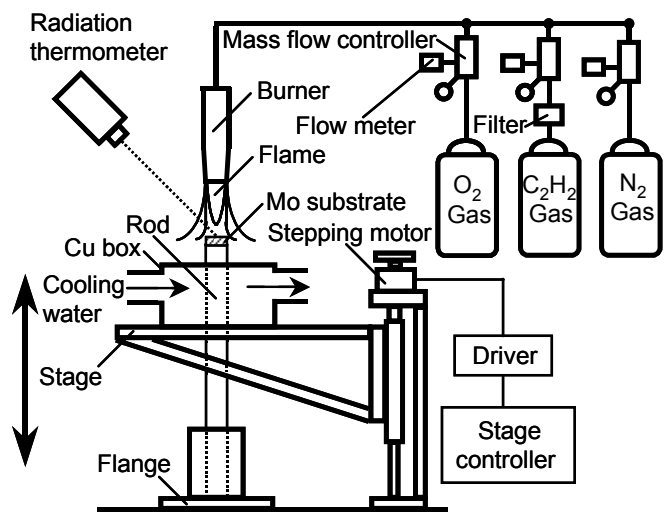

Fig. 1. Experimental setup for synthesizing diamond by flame combustion.
Table 1 Conditions for diamond syntheses.

\begin{tabular}{l|c}
\hline \hline Reaction gas & $\mathrm{C}_{2} \mathrm{H}_{2}+\mathrm{O}_{2}+\mathrm{N}_{2}$ \\
\hline Film surface temperature & $1223 \sim 1423[\mathrm{~K}]$ \\
\hline Pressure & $10^{5}[\mathrm{~Pa}]$ \\
\hline Substrate surface roughness, $R_{\mathrm{a}}$ & $0.18[\mu \mathrm{m}]$ \\
\hline $\begin{array}{l}\text { Inner cone-to-substrate } \\
\text { distance }\end{array}$ & $1.5[\mathrm{~mm}]$ \\
\hline $\mathrm{C}_{2} \mathrm{H}_{2}$ Flow rate, $F_{\mathrm{a}}$ & $70.9\left[\mathrm{~cm}^{3} / \mathrm{s}\right]$ \\
\hline $\mathrm{O}_{2}$ Flow rate, $F_{\mathrm{o}}$ & $63.8\left[\mathrm{~cm}^{3} / \mathrm{s}\right]$ \\
\hline Flow fraction, $R_{\mathrm{f}}=F_{\mathrm{o}} / F_{\mathrm{a}}$ & 0.90 \\
\hline
\end{tabular}

Table 2 Conditions of nitrogen addition.

\begin{tabular}{c|c|c}
\hline \hline $\begin{array}{c}\text { Commercial } \mathrm{C}_{2} \mathrm{H}_{2} \\
+\mathrm{O}_{2}+\mathrm{N}_{2}\end{array}$ & $\begin{array}{c}\text { High-purity } \mathrm{C}_{2} \mathrm{H}_{2} \\
+\mathrm{O}_{2}+\mathrm{N}_{2}\end{array}$ & $\begin{array}{c}\mathrm{N}_{2} \text { flow rate } \\
F_{\mathrm{n}}\left[\mathrm{cm}^{3} / \mathrm{s}\right]\end{array}$ \\
\hline Case 1 & Case 4 & 0.000 \\
\hline Case 2 & Case 5 & 0.500 \\
\hline Case 3 & Case 6 & 1.000 \\
\hline
\end{tabular}

three-step method was $30 \mu \mathrm{m}$ per hour.

\subsection{Synthetic conditions}

The synthetic conditions are shown in Table 1. We previously determined these conditions $^{5,6}$, which are optimal for preventing delamination during the synthesis of diamond films. In this study, to obtain nanocrystalline diamond films, commercial acetylene or high-purity acetylene was used as fuel for the synthesis. Here, oxygen was used as the fuel for the synthesis. Moreover, nitrogen gas was added as the nanocrystalline diamond promotion agent, nitrogen flow rate was varied as Table 2 . The synthetic time for each of the three steps in the method used was set to $1200 \mathrm{~s}$, and the total synthesis time of all three steps was set to $1 \mathrm{~h}^{5,6}$.

\section{Experimental results and discussion}

\subsection{Effects of nitrogen flow rate on delamination}

The delamination of the synthesized films in all cases was successfully prevented at a very high probability. In this regard, the following reasons are given. The films were synthesized using the three-step synthesis method, which is effective in preventing delamination. Moreover, films were synthesized under the previously determined optimal conditions $^{5,6}$ to prevent delamination.

\subsection{Investigation of synthesized films using commercial acetylene gas}

The films were analyzed by scanning electron microscopy (SEM: KEYENCE VE-9800) and X-ray diffraction (XRD: JEOL JDX- 3530) analysis.

SEM images of the films synthesized with varying the nitrogen flow rate using the 

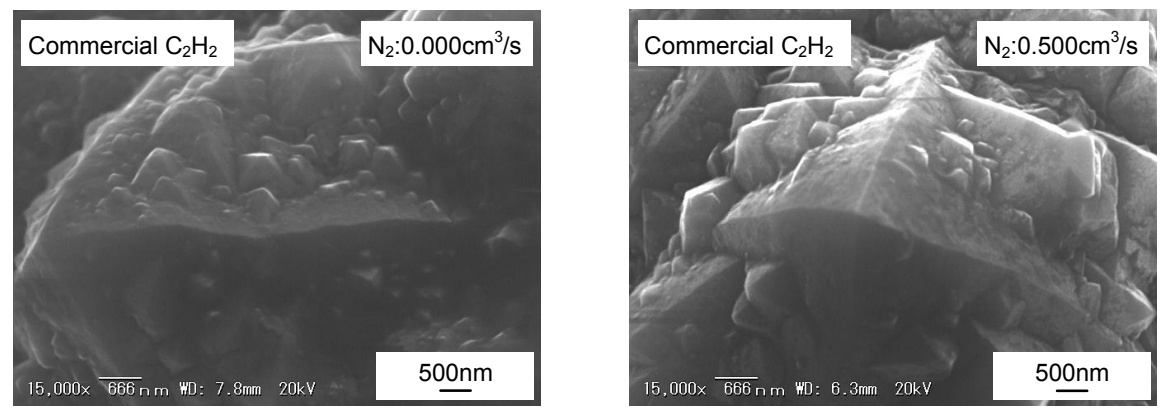

Fig. 2. SEM image of the synthesized film in Case 1. Fig. 3. SEM image of the synthesized film in Case 2
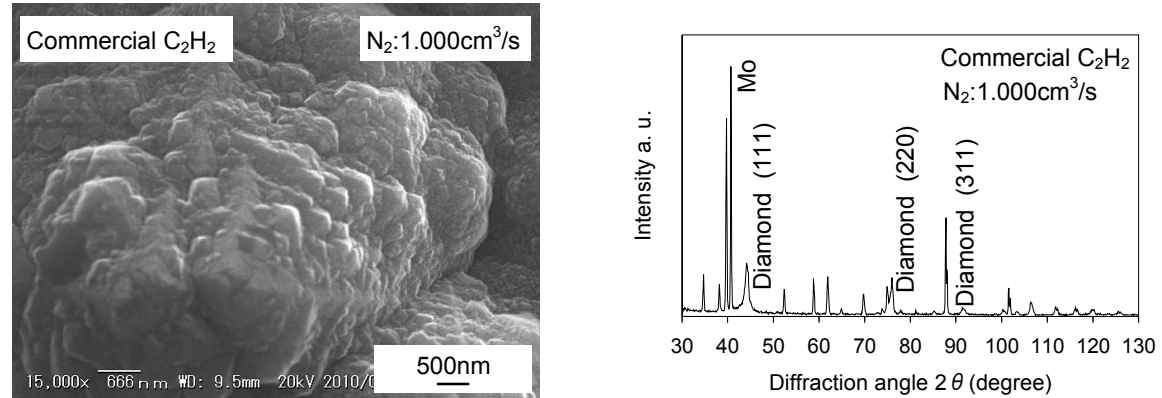

Fig. 4. SEM image of the synthesized film in Case 3. Fig. 5. XRD patterns of the synthesized film in Case 3 .

mixture of commercial acetylene and oxygen gas are shown in Figs. 2, 3 and 4. Figs. 2, 3 and 4 show the films synthesized in Cases 1, 2 and 3, respectively. In the SEM image in Fig. 2, the microcrystallites was synthesized. They showed an octahedral morphology. The diamond nanocrystallites was synthesized partially on the diamond microcrystallites. From the SEM image in Fig. 3, the diamond nanocrystallites was synthesized on the microcrystallites. The nanocrystallites shown here could be slightly increased compared with those in Case 1 (without nitrogen addition). In the SEM image in Fig. 4, the nanocrystallites could be easily synthesized compared with the crystallites in Case 2, and diamond nanocrystallites could still grow. They also showed almost uniform size and high density on the microcrystallites. Typical XRD pattern of Case 3 is shown in Fig. 5. In the XRD patterns in Fig. 5, peaks showing the existence of diamond (111), (220) and (311) surfaces were confirmed. The diamond crystallites was synthesized.

\subsection{Investigation of synthesized films using high-purity acetylene gas}

SEM images of the films synthesized with varying the nitrogen flow rate using the mixture of high-purity acetylene and oxygen gas are shown in Figs. 6, 7 and 8. Figs. 6, 7 and 8 show the films synthesized in Cases 4, 5 and 6, respectively. In the SEM image in Fig. 6, the diamond nanocrystallites was not synthesized. The synthesized crystallites did not develop diamond morphology. From the SEM image in Fig. 7, the microcrystallites was synthesized, and the diamond nanocrystallites was synthesized continuously on the microcrystallites. They showed an octahedral morphology. Moreover, they showed 

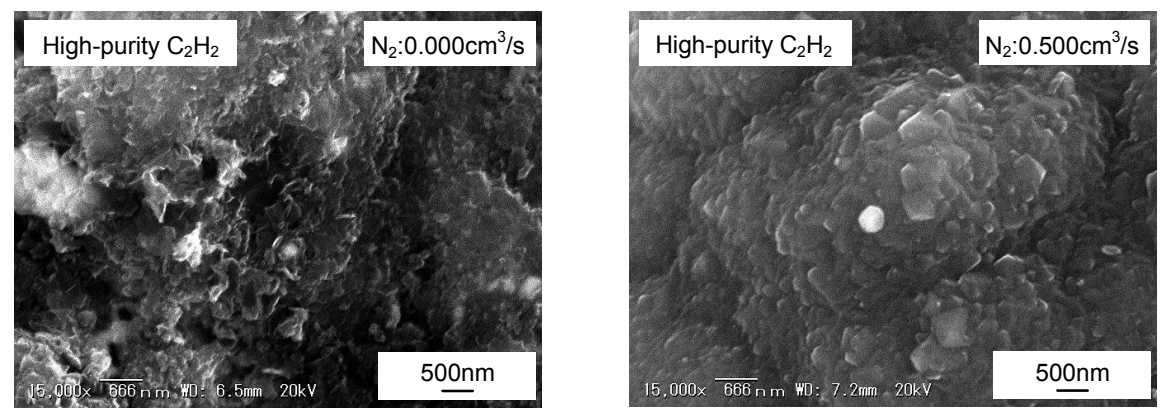

Fig. 6. SEM image of the synthesized film in Case 4.

Fig. 7. SEM image of the synthesized film in Case 5.
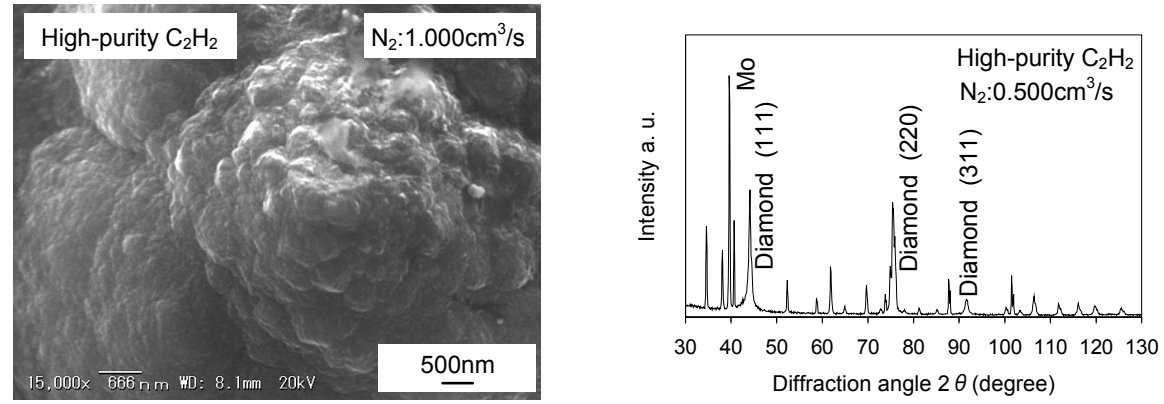

Fig. 8. SEM image of the synthesized film in Case 6. Fig. 9. XRD patterns of the synthesized film in Case 5.

uniform size and very high density. The optimal amount of nitrogen added promoted the growth of nanocrystallites of uniform size and very high density. In the SEM image in Fig. 8, the synthesized nanocrystallites could not be easily etched compared with the crystallites in Case 5, and the synthesized crystallites showed high density. Typical XRD pattern of Case 5 is shown in Fig. 9. In the XRD pattern in Fig. 9, it is shown that, peaks showing the existence of diamond (111), (220) and (311) surfaces were clearly confirmed; the peak of the diamond (111) surface was distinct. In the SEM and XRD results, the synthesized diamond nanocrystallites in Case 5 showed an almost octahedral morphology, uniform size and very high density. In this experiment, the optimal nitrogen flow rate was found to be $0.500 \mathrm{~cm}^{3} / \mathrm{s}$.

\subsection{Effects of nitrogen addition on the properties of synthesized diamond films}

Nitrogen addition to commercial or high purity acetylene affects nanocrystalline diamond synthesis. Moreover, the crystallite morphology of synthesized diamond films can be changed by varying the nitrogen flow rate. We considered that the reason in results is the difference between the components of commercial and high-purity acetylene gas. Several researchers have shown that nitrogen addition affects the morphology of synthesized diamond crystallites ${ }^{1,2,7,8}$. Therefore, in this study, nitrogen gas was added to synthesize diamond nanocrystallites films. The diamond morphology of the synthesized films was improved by adding an optimal amount of nitrogen. Consequently, nitrogen is a very important factor for diamond nanocrystallites synthesis. Here, Commercial acetylene is 
the variable purity $(91.5 \sim 98.5 \%)$ as a result of dissolved impurities. High-purity acetylene was obtained through special dissolution of pure acetylene of stable and high purity (more than $99.5 \%)^{9}$. And, the amount of nitrogen in high-purity acetylene was investigated and found to be $0.01 \sim 0.17 \%$, which was negligible. However, the amount of nitrogen in commercial acetylene is $0.8 \sim 2.5 \%{ }^{9}$. From results, the diamond morphology and density of the synthesized diamond films was changed by varying the nitrogen flow rate. The reason is difference in nitrogen amount between high-purity acetylene and commercial acetylene. And, we considered that the reason was that $\mathrm{CN}$-radicals in the acetylene feather being affected.

\section{Conclusions}

In this study, to obtain nanocrystalline diamond films and to achieve good adhesion, diamond films were synthesized by flame combustion using a mixture of commercial or high-purity acetylene and oxygen gas with the addition of nitrogen gas, and nitrogen flow rate was varied. The delamination of the films synthesized with varying the nitrogen flow rate was successfully prevented at a very high probability. In the case of the films synthesized with varying the nitrogen flow rate using the mixture of commercial acetylene and oxygen gas, when nitrogen gas was increased, the diamond nanocrystallites was high density on the microcrystallites. From the results of the films synthesized with varying the nitrogen flow rate using the mixture of high-purity acetylene and oxygen gas, as the optimal amount of nitrogen added, the diamond nanocrystallites was synthesized continuously on the microcrystallites. They had an octahedral morphology of uniform size and high density.

\section{Acknowledgments}

This work was supported by Grant-in-Aid for Scientific Research (C) (21560075) from the Ministry of education, Science, Sports and Culture of Japan.

\section{References}

1. C. J. Tang, A. J. Neves, S. Pereira, A. J. S. Fernandes, J. Gracio and M. C. Carmo: Diamond and Related Materials, 17-1 (2008) 72-78.

2. H. Lee, H. Li, H. Jeon and W. Lee: Diamond and Related Materials, 19-11 (2010) 1393-1400.

3. Y. Hirose, N. Okada and H. Koike: J. Combustion Society of Japan, 80 (1989) 1-17.

4. M. Takahashi, O. Kamiya and T. Ohyoshi: Int. J. Modern physics B, 20-25, 26 and 27 (2006) 3926-3931.

5. M. Takahashi, O. Kamiya and T. Ohyoshi: J. Sol. Mech. and Mate. Eng., 1-2 (2007) 223-231.

6. M. Takahashi, Y. Harada, O. Kamiya and T. Ohyoshi: J. Sol. Mech. and Mate. Eng., 3-6 (2009) 853-864.

7. S. T. Kshirsagar, R. B. Kshirsagar, P. S. Patil, A. V. Kulkarni, A. B. Mandale, A. B. Gaikwad and S. P. Gokhale: Diamond and Related Materials, 14-2 (2005) 232-242.

8. M. A. Neto and E. Pereira: Diamond and Related Materials, 15-4-8 (2006) 465-471.

9. G. Saito, Y. Saito, T. Nakamura and S. Abe: Bunseki Kagaku, 37-10 (1988) T100-104. 